\title{
Polynomial sequences of binomial-type arising in graph theory
}

\author{
Jonathan Schneider \\ Department of Computer Science \\ Princeton University, Princeton, NJ 08540, U.S.A. \\ js44@princeton.edu
}

Submitted: Sep 8, 2013; Accepted: Feb 8, 2014; Published: Feb 28, 2014

Mathematics Subject Classifications: 05A40, 05C31

\begin{abstract}
In this paper, we show that the solution to a large class of "tiling" problems is given by a polynomial sequence of binomial type. More specifically, we show that the number of ways to place a fixed set of polyominos on an $n \times n$ toroidal chessboard such that no two polyominos overlap is eventually a polynomial in $n$, and that certain sets of these polynomials satisfy binomial-type recurrences. We exhibit generalizations of this theorem to higher dimensions and other lattices. Finally, we apply the techniques developed in this paper to resolve an open question about the structure of coefficients of chromatic polynomials of certain grid graphs (namely that they also satisfy a binomial-type recurrence).
\end{abstract}

\section{Introduction}

A sequence $p_{0}=1, p_{1}, p_{2}, \ldots$ of polynomials is a polynomial sequence of binomial type if it satisfies the identity

$$
\left(\sum_{i=0}^{\infty} p_{i}(1) x^{i}\right)^{n}=\sum_{i=0}^{\infty} p_{i}(n) x^{i} .
$$

Binomial-type sequences were introduced by Rota, Kahaner, and Odlyzko in 1975 [10] and play an important role in the theory of umbral calculus. Outside of the context of umbral calculus, polynomial sequences of binomial type possess the useful property that they are completely determined by the sequence of their values when evaluated at a single point. Several important polynomial sequences, such as the Abel polynomials and the Touchard polynomials, are of binomial type.

In this paper we demonstrate that polynomial sequences of binomial type arise from a large class of problems occurring in graph theory. In particular, these sequences occur 
in problems where we wish to enumerate the number of ways to place some objects on a "toroidal" periodic structure such that no two overlap. One simple example of this phenomenon is the following. Take an $n \times n$ chessboard and identify opposite edges to make it toroidal. If we let $p_{k}\left(n^{2}\right)$ equal the number of ways to place $k$ dominoes on this grid (aligned with the grid's edges) such that no two dominoes overlap, then it turns out that, for sufficiently large $n$ the values $p_{k}\left(n^{2}\right)$ are given by a polynomial in $n^{2}$. Moreover, this sequence of polynomials (viewed as a sequence in $k$ ) is a polynomial sequence of binomial type.

Our methods allow us to easily generalize these results. The main result of our paper is a generalization of the above phenomenon to arbitrary sets of polyominos on toroidal grids of any dimension. We also demonstrate some interesting further generalizations of this result; for example, we show that the same binomial-type relation holds when we can assign arbitrary integer weights to polyominos and then count placements that have a total weight of $k$. In addition, we show that there is a very natural continuous analogue of these results concerning placing arbitrary bounded measurable 'shapes' in a continuous $d$-dimensional torus.

Finally, we apply these results to provide a proof of an open problem due to Stanley [9] concerning coefficients of the chromatic polynomial $\chi_{n}(x)$ of the two-dimensional toroidal grid graph. Much previous research into the chromatic polynomial $\chi_{n}(x)$ of toroidal grid graphs focused primarily on the asymptotics of this polynomial, particularly the limit $\lim _{n \rightarrow \infty}\left(\chi_{n}(x)\right)^{1 / n^{d}}$ (see, for instance $\left.[1,2,3,4,5,6,7,8]\right)$. For example, it is known that for $d=2$ and $x=3$, this limit is equal to $(4 / 3)^{3 / 2}$.

The open problem due to Stanley asks to show that the coefficient of $x^{n^{2}-k}$ in $\chi_{n}(x)$ is (up to sign) for sufficiently large $n$ a polynomial in $n^{2}$, and that the polynomials for different $k$ form a polynomial sequence of binomial type. By using Whitney's brokencircuit theorem to reduce this to an overlap problem of the style above, we resolve this open problem (and in fact, provide a generalization that holds for chromatic polynomials of toroidal grid graphs of any positive dimension).

Our paper is structured as follows. In Section 2, we define some terminology that we use throughout this paper. In Section 3, we prove that the problem of enumerating the number of non-overlapping placements does in fact give rise to a polynomial for sufficiently large $n$. We additionally show how to write these polynomials in a nice form reminiscent of certain generating functions. In Section 4, we introduce the notion of an intersection schema and use it to prove our main theorem. In Section 5, we discuss generalizations of our main result to the cases of assigning integer weights of polyominos, continuous tori, non-toroidal grids, and other types of lattices. Finally, in Section 6, we apply our main result along with Whitney's broken-circuit theorem to solve the open problem mentioned above.

\section{Background and Definitions}

We begin with some graph-theoretic notation. We let $C_{n}$ denote the cycle on $n$ vertices, $P_{n}$ denote the path on $n$ vertices, and $C_{\infty}$ denote the doubly infinite path. 
Definition 1. Given two graphs $G_{1}$ and $G_{2}$, we define the product graph $G_{1} \times G_{2}$ as follows. The vertices of $G_{1} \times G_{2}$ are given by ordered pairs $\left(v_{1}, v_{2}\right)$ where $v_{1} \in G_{1}$ and $v_{2} \in G_{2}$. The two vertices $\left(v_{1}, v_{2}\right)$ and $\left(v_{1}^{\prime}, v_{2}^{\prime}\right)$ are adjacent if either $v_{1}=v_{1}^{\prime}$ and $v_{2}$ is adjacent to $v_{2}^{\prime}$ in $G_{2}$ or $v_{2}=v_{2}^{\prime}$ and $v_{1}$ is adjacent to $v_{1}^{\prime}$ in $G_{1}$. We write $G^{r}$ for the expression $G \times G \times \cdots \times G$ (with $r$ copies of $G$ ).

Definition 2. The d-dimensional toroidal grid graph of size $n, T_{n}^{d}$, is the graph $\left(C_{n}\right)^{d}$. Similarly, the $d$-dimensional infinite toroidal grid graph $T_{\infty}^{d}$ is the graph $\left(C_{\infty}\right)^{d}$, and the $d$-dimensional grid graph of size $n, L_{n}^{d}$, is the graph $\left(P_{n}\right)^{d}$.

Definition 3. A d-dimensional figure is a finite subset of vertices of $T_{\infty}^{d}$, up to translation. That is, two figures are considered equivalent if we can get from one to the other by adding a fixed integer vector to the coordinates of each of its vertices. We say a figure is of size $s$ if it contains $s$ vertices. We also say a figure is of girth $g$ if the maximum $\ell_{\infty}$ norm between two vertices in the figure is equal to $\mathrm{g}$.

We have defined figures above as subsets of $T_{\infty}^{d}$. However, it is clear that, for any specific figure, if $n$ is large enough (in particular, larger than the girth of the figure), then we can also view the figure as a subset of $T_{n}^{d}$ (up to translation). We will often abuse notation in this way by talking about "placing" figures on $T_{n}^{d}$. In such cases, we will always assume that we are taking $n$ large enough so that this makes sense.

We next define what it means for a sequence of polynomials to be of binomial type.

Definition 4. The sequence of polynomials $\left\{p_{i}(n)\right\}_{i \geqslant 0}$ is of binomial-type if it satisfies the following three properties: i. $p_{0}(n)=1$, ii. $p_{i}(0)=0$ for $i>0$, and iii. the identity given by equation (2) holds for all nonnegative $n$.

$$
\left(\sum_{i=0}^{\infty} p_{i}(1) x^{i}\right)^{n}=\sum_{i=0}^{\infty} p_{i}(n) x^{i}
$$

An equivalent reformulation of our third condition is that the identity given by equation (3) holds for all nonnegative $n$.

$$
p_{n}(x+y)=\sum_{i=0}^{n} p_{i}(x) p_{n-i}(y)
$$

Our definition of binomial-type differs slightly from the definition most often found in the literature, where equation (3) contains an additional factor of $\left(\begin{array}{c}n \\ i\end{array}\right)$. The two definitions are easily interchangeable, however; if $\left\{p_{i}(n)\right\}$ is a sequence of binomial-type under our definition, then $\left\{i ! p_{i}(n)\right\}$ is a sequence of binomial-type under the traditional definition.

\section{Polynomiality}

In this section, we demonstrate that several sequences related to the number of ways to place a fixed set of figures on a lattice are eventually described by polynomials. More specifically, we have the following main result. 
Theorem 5. Let $S$ be a finite multiset of d-dimensional figures. Let $f_{S}(n)$ be the number of ways to place all of the figures in $S$ on $T_{n}^{d}$ with no overlap (for a finite set of small values of $n$, there may be figures that are impossible to place on $T_{n}^{d}$; in this case, let $\left.f_{S}(n)=0\right)$. Then there exists a positive integer $n_{0}$ and an integer polynomial $p(x)$ such that $f_{S}(n)=p\left(n^{d}\right)$ for all $n \geqslant n_{0}$.

Since there is some subtlety in dealing with multisets containing repeated indistinguishable figures, in the first half of this section (Subsection 3.1) we prove this result only for sets of distinct figures. In the second half (Subsection 3.2), we generalize to the case where repeats of figures are allowed.

\subsection{Without repeats}

In this subsection, we prove Theorem 5 for the case where $S$ contains no repeated figures. In particular, we prove the following simpler result.

Theorem 6. Let $S$ be a finite set of distinct d-dimensional figures. Let $f_{S}(n)$ be the number of ways to place all of the figures in $S$ on $T_{n}^{d}$ such that none overlap (for a finite set of small values of $n$, there may be figures that are impossible to place on $T_{n}^{d}$; in this case, let $\left.f_{S}(n)=0\right)$. Then there exists a positive integer $n_{0}$ and an integer polynomial $p(x)$ such that $f_{S}(n)=p\left(n^{d}\right)$ for all $n \geqslant n_{0}$.

Throughout this subsection and the next, we will repeatedly make use of the following notion of an overlap graph.

Definition 7. An overlap graph is a graph $G$ whose vertices are labelled by $d$-dimensional figures (for some $d$ ). A placement of these figures on $T_{n}^{d}$ (or $T_{\infty}^{d}$ ) is consistent with $G$ if, whenever figures $f_{1}$ and $f_{2}$ are adjacent in $G$, they overlap in $T_{n}^{d}\left(\right.$ or $T_{\infty}^{d}$ ).

Note that if a placement of figures is consistent with an overlap graph, so are all translations of this placement of figures. This inspires the following definition.

Definition 8. A configuration of $d$-dimensional figures is an equivalence class of placements of $d$-dimensional figures on $T_{n}^{d}$ (or $T_{\infty}^{d}$ ) where two configurations are equivalent if they are translations of each other in $T_{n}^{d}\left(\right.$ or $\left.T_{\infty}^{d}\right)$. A configuration $c$ is consistent with a graph $G$ if all of its placements are consistent with $G$; in this case, we write $c \unlhd G$.

If $f_{1}$ and $f_{2}$ are not adjacent in $G$, they may or may not overlap in $T_{n}^{d}$ (or $T_{\infty}^{d}$ ); only one direction of the above implication holds. In addition, for now we will assume that the vertices of our overlap graphs are labelled with distinct $d$-dimensional figures; we will lift this constraint in the following subsection.

We next prove three useful lemmas about overlap graphs.

Lemma 9. If an overlap graph $G$ is connected, then, there are only finitely many configurations of these figures on $T_{\infty}^{d}$ consistent with $G$. We call this number $v(G)$. 
Proof. Since $G$ is connected, there exists a path of edges of $G$ between any two vertices of $G$. This implies that, for any two figures $f_{1}$ and $f_{2}$ in a consistent placement of these figures on $T_{\infty}^{d}$, we can construct a sequence of figures starting at $f_{1}$ and ending at $f_{2}$ such that each figure intersects the next figure in the sequence.

Now, since each of the figures has finite size, and since there are a finite number of figures, this implies that the maximum distance (along edges of the graph) between any two points belonging to figures in our placement is bounded. Since there are only finitely many ways to place a finite number of figures in a bounded region of $T_{\infty}^{d}$, this establishes that $v(G)$ is finite.

Lemma 10. Let $G$ be a connected overlap graph. Then the number of placements of figures on $T_{n}^{d}$ consistent with $G$ is equal to $v(G) n^{d}$ for sufficiently large $n$.

Proof. By Lemma 9, we know that there are $v(G)$ distinct consistent configurations of these figures on $T_{\infty}^{d}$. For sufficiently large $n$, it will be possible to embed each of these $v(G)$ configurations in $T_{n}^{d}$. Finally, for each choice of consistent configuration, there are $n^{d}$ possible translations in $T_{n}^{d}$. Therefore, there are a total of $v(G) n^{d}$ consistent placements on $T_{n}^{d}$ for sufficiently large $n$.

Lemma 11. Let $G$ be an overlap graph with connected components $G_{1}, G_{2}, \ldots, G_{r}$. Then, the number of placements of figures on $T_{n}^{d}$ consistent with $G$ is equal to $v\left(G_{1}\right) \ldots v\left(G_{r}\right) n^{r d}$.

Proof. Note that we can place all the connected components of $G$ independently. Since by Lemma 10, there are $v\left(G_{i}\right) n^{d}$ placements on $T_{n}^{d}$ consistent with $G_{i}$ (for sufficiently large $n$ ), overall there will be

$$
\prod_{i=1}^{r} v\left(G_{i}\right) n^{d}=\left(\prod_{i=1}^{r} v\left(G_{i}\right)\right) n^{r d}
$$

placements of figures on $T_{n}^{d}$ consistent with $G$ (for sufficiently large $n$ ), as desired.

With these lemmas, the proof of Theorem 6 reduces to a straightforward application of the principle of inclusion-exclusion.

Proof of Theorem 6. Label the figures in $S$ as $f_{1}, f_{2}, \ldots, f_{m}$. We wish to count the number of placements of these figures on $T_{n}^{d}$ such that no two figures overlap. Thus, for each pair $1 \leqslant i<j \leqslant m$, let $E(i, j)$ be the set of placements of these figures where $f_{i}$ and $f_{j}$ intersect, and let $U$ be the set of all placements of these figures. For convenience of notation, let $\mathcal{P}$ be the set of all pairs $(i, j)$ where $1 \leqslant i<j \leqslant m$; if $p=(i, j)$, we will also let $E(p)$ stand for $E(i, j)$. Finally, let $U$ be the set of all possible placements of these figures on $T_{n}^{d}$; equivalently, the number of placements consistent with the overlap graph $G$ containing no edges. Note that, for sufficiently large $n,|U|=\left(n^{d}\right)^{m}$, since there are at $n^{d}$ ways to place each figure.

Then, by the principle of inclusion-exclusion, the number of placements where no two figures overlap is equal to 


$$
|U|-\sum_{p_{1} \in \mathcal{P}}\left|E\left(p_{1}\right)\right|+\sum_{p_{1}, p_{2} \in \mathcal{P}}\left|E\left(p_{1}\right) \cap E\left(p_{2}\right)\right|-\sum_{p_{1}, p_{2}, p_{3} \in \mathcal{P}}\left|E\left(p_{1}\right) \cap E\left(p_{2}\right) \cap E\left(p_{3}\right)\right|+\cdots .
$$

To show that this expression is eventually a polynomial in $n^{d}$, it suffices to show that each of the individual terms is eventually a polynomial in $n^{d}$. Now, $\mid E\left(p_{1}\right) \cap E\left(p_{2}\right) \cap \cdots \cap$ $E\left(p_{k}\right)|=| E\left(i_{1}, j_{1}\right) \cap E\left(i_{2}, j_{2}\right) \cap \cdots \cap E\left(i_{k}, j_{k}\right) \mid$ is equal to the number of placements where figure $f_{i_{r}}$ intersects figure $f_{j_{r}}$ for each $r$ between 1 and $k$. But this is simply equal to the number of placements consistent with the overlap graph $G$ which contains an edge between $f_{i_{r}}$ and $f_{j_{r}}$ for each $r$ between 1 and $k$. By Lemma 11, this is eventually a polynomial in $n^{d}$. This concludes the proof.

For a connected overlap graph $G$, let $a(G)=(-1)^{|E|} v(G)$. By substituting values into Equation 5 from Lemma 11, we have the following corollary.

Corollary 12. Let $N=n^{d}$, and let $m$ be the number of figures in $S$. Then, for sufficiently large $n$, the function $f_{S}(n)$ defined in Theorem 6 is equal to

$$
\sum_{r=1}^{m} \sum \frac{1}{r !} a\left(g_{1}\right) a\left(g_{2}\right) \cdots a\left(g_{r}\right) N^{r}
$$

where the inner sum runs over all ordered $r$-tuples of connected overlap graphs that union to an overlap graph for the set $S$ (equivalently, the union of the sets of figures corresponding to the vertices of the $g_{i}$ is equal to the set $S$ ).

Note that since $S$ contains only distinct figures, we could easily remove the factor of $1 / r$ ! in equation (6) and instead sum over all unordered $r$-tuples. However, for reasons to be explained in Section 4, it is more convenient to write our polynomial in this form.

\subsection{With repeats}

In the previous section, we proved Theorem 5 for the specific case where $S$ contained no repeated indistinguishable figures. In the case that we have several of the same figure, certain details in the above proof (in particular, Lemma 11) fail to hold. For example, if our set $S$ contains two identical figures, the number of total possible placements is neither $n^{2 d}$ nor $n^{2 d} / 2$, but rather $\left(n^{2 d}+n^{d}\right) / 2$; the extra $n^{d}$ term arises from the fact that our two indistinguishable figures can occupy exactly the same location.

However, if we have repeated distinguishable figures, all of the logic in the previous section continues to hold. This observation gives rise to a simple proof of Theorem 5 .

Proof of Theorem 5. Assume that $S$ contains $c_{i}$ copies of figure $f_{i}$, for each $1 \leqslant i \leqslant m$.

For each group of indistinguishable repeated figures in $S$, "color" them to make them distinguishable. Then the proof of Theorem 6 implies that the number of ways to place these figures on $T_{n}^{d}$ such that no two figures overlap is eventually some polynomial $p\left(n^{d}\right)$ for large enough $n$. 
But now, if we ignore the different colors, each configuration where no two figures overlap is counted exactly $c_{1} ! c_{2} ! \ldots c_{m}$ ! times. Therefore in the case of indistinguishable repeated figures, the number of placements of these figures such that no two figures overlap is eventually $p\left(n^{d}\right) /\left(c_{1} ! c_{2} ! \ldots c_{m} !\right)$, which is also a polynomial in $n^{d}$.

For reasons that will be explained in the next section, we would also like to write this polynomial in the same form as equation (6). In order to construct the correct function $a(g)$, we must introduce some more notation.

Definition 13. For any configuration $c$, we can partition the figures of $c$ into $k$ maximal sets $O_{i}$ such that all the figures in $O_{i}$ are identical and overlap completely. Then the weight $w_{c}$ of a configuration $c$ is defined to equal $\prod_{i=1}^{k}\left(o_{i} !\right)^{-1}$, where $o_{i}=\left|O_{i}\right|$.

Definition 14. In a connected overlap graph $G$, assume that there are a total of $c_{i}$ (vertices labeled with) figures of type $f_{i}$ for each $1 \leqslant i \leqslant m$. Then we define $\alpha(G)=$ $|\operatorname{Aut}(G)|^{-1} \prod_{i=1}^{m} c_{i}$ !, where $\operatorname{Aut}(G)$ is the group of automorphisms of the graph $G$ that preserve labelling (that is, they send vertices labelled with figures of type $f_{i}$ to vertices labelled with figures of type $f_{i}$ ).

Note that if we let $H$ be the group of all permutations of the vertices of $G$ which send figures of type $f_{i}$ to figures of type $f_{i}$, then $\alpha(G)$ can be equivalently defined as $|H| /|\operatorname{Aut}(G)|$. Similarly, this is just the number of non-isomorphic ways to color each set of $c_{i}$ figures of type $f_{i}$ with $c_{i}$ distinguishable colors. We will make use of this fact in the proof of the following theorem.

Theorem 15. For a connected overlap graph g, define

$$
a(g)=(-1)^{|E|} \alpha(g) \sum_{c \unlhd g} w_{c}
$$

where the sum runs over all configurations c consistent with $g$. Then, as before, we have that

$$
f_{S}(n)=\sum_{r=1}^{m} \sum \frac{1}{r !} a\left(g_{1}\right) a\left(g_{2}\right) \cdots a\left(g_{r}\right) N^{r}
$$

where the inner sum runs over all ordered $r$-tuples of connected overlap graphs that union to an overlap graph for the set $S$.

Proof. Let $S$ contain $\mu$ different types of figures, with $c_{i}$ figures of type $f_{i}$ for each $i$ between 1 and $\mu$ (so $\sum c_{i}=m$ ). Our goal will be to show that the right hand side of formula (8) is exactly

$$
\frac{f_{\bar{S}}(n)}{\prod_{i=1}^{\mu} c_{i} !},
$$

where $\bar{S}$ is the set obtained by coloring all the figures in $S$ so that they are distinguishable; it then follows from the above proof of Theorem 5 that this is equal to $f_{S}(n)$, as desired. 
Let $p$ be any placement of the figures of $S$ onto $T_{n}^{d}$. We will show that the number of times $p$ is counted in the right hand side of formula (8) is equal to the number of times it is counted in formula (9).

To do this, first note that by the definition of $\alpha(G)$, for any connected overlap graph $g$, we can write $a(g)=\sum a^{\prime}(h)$, where the sum runs over all graphs $h$ obtained by coloring all the figures of type $f_{i}$ distinguishably (note that there are $\alpha(g)$ such graphs). Our new function $\alpha^{\prime}(h)$ is now given just by $\alpha^{\prime}(h)=(-1)^{|E|} \sum_{c \triangleleft h} w_{c}$ (where for a configuration $c$ to be consistent with $h$, it simply has to be consistent with the original graph $g$ ). We can therefore write $f_{S}(n)$ as

$$
f_{S}(n)=\sum_{r=1}^{m} \sum \frac{1}{r !} a^{\prime}\left(h_{1}\right) a^{\prime}\left(h_{2}\right) \cdots a^{\prime}\left(h_{r}\right) N^{r}
$$

where this new sum runs over ordered $r$-tuples of these additionally colored overlap graphs $h_{i}$.

The terms of this sum that contribute to the total count for our placement $p$ are indexed by a choice of additionally colored overlap graphs $h_{i}$ and partitions of $p$ into $r$ subplacements $p_{i}$ such that each $p_{i}$ is consistent with $h_{i}$. We can write one such term as

$$
t(\mathbf{h}, \mathbf{p})=\frac{1}{r !}(-1)^{\sum\left|E_{i}\right|} w_{p_{1}} w_{p_{2}} \cdots w_{p_{r}}
$$

where $w_{p_{i}}$ is the weight of placement $p_{i}$ (which is the same as the weight of the configuration $c_{i}$ to which $p_{i}$ belongs), and $\mathbf{h}$ and $\mathbf{p}$ represent the two $r$-tuples $\left(h_{1}, h_{2}, \ldots, h_{r}\right)$ and $\left(p_{1}, p_{2}, \ldots, p_{r}\right)$ respectively.

Now, as in Definition 13, partition $S$ into $k$ maximal sets $O_{i}$ such that all the figures in $O_{i}$ are identical and overlap completely in $p$ (and let $o_{i}=\left|O_{i}\right|$ ). Note that the overlap sets $O_{i}$ for placement $p$ can be further partitioned among the $r$ subplacements that union to $p$. Therefore, for each $1 \leqslant j \leqslant r$, let $O_{i j}$ be the subset of $O_{i}$ that belongs to subplacement $p_{j}$, and let $o_{i j}=\left|O_{i j}\right|$. Note then that $w_{p_{i}}=\left(o_{1 i} ! o_{2 i} ! \ldots o_{k i} !\right)^{-1}$, and thus that

$$
t(\mathbf{h}, \mathbf{p})=\frac{1}{r !}(-1)^{\sum\left|E_{i}\right|} \prod_{i=1}^{k} \prod_{j=1}^{r}\left(o_{i j} !\right)^{-1} .
$$

Let us now count the number of times placement $p$ is counted in $f_{\bar{S}}(n)$. Let $\bar{p}$ be a placement of the (colored) figures of $\bar{S}$ such that, when color is ignored, is equal to $p$. We will say that $\bar{p}$ decolors to $p$. By Corollary 6 and as per the logic above, for each choice of overlap graphs $h_{i}$ and each partition of $\bar{p}$ into $r$ subplacements $\bar{p}_{i}$ (such that $\bar{p}_{i}$ is consistent with $h_{i}$ ), we contribute

$$
t(\mathbf{h}, \overline{\mathbf{p}})=\frac{1}{r !}(-1)^{\sum\left|E_{i}\right|}
$$

towards the overall count for placement $\bar{p}$.

Now, consider the number of choices of $\overline{\mathbf{p}}$ such that $\bar{p}$ decolors to $p$ and $\bar{p}_{i}$ decolors to $p_{i}$ for each $i$ between 1 and $r$. We would like the sum of $t(\mathbf{h}, \overline{\mathbf{p}})$ over these choices to 
equal $\left(\prod_{i=1}^{\mu} c_{i} !\right) t(\mathbf{h}, \mathbf{p})$, as this would imply that placement $p$ is accounted for a multiple of $\prod_{i=1}^{\mu} c_{i}$ ! more times in $f_{\bar{S}}(n)$ than in $f_{S}(n)$. From formulas (12) and (13), this occurs if the number of such choices equals

$$
\left(\prod_{i=1}^{\mu} c_{i} !\right)\left(\prod_{i=1}^{k} \prod_{j=1}^{r}\left(o_{i j} !\right)^{-1}\right)
$$

We can rewrite this as

$$
\left(\frac{\prod_{i=1}^{\mu} c_{i} !}{\prod_{i=1}^{k} o_{i} !}\right)\left(\prod_{i=1}^{k} o_{i} ! \prod_{j=1}^{r}\left(o_{i j} !\right)^{-1}\right)
$$

The first term, $\prod_{i=1}^{\mu} c_{i} ! / \prod_{i=1}^{k} o_{i} !$, counts the number of ways to, for each $i$ between 1 and $\mu$, assign $c_{i}$ distinct colors to all the sets $O_{i}$ containing figures of type $f_{i}$. The second term, $o_{i} ! \prod_{j=1}^{r}\left(o_{i j} !\right)^{-1}$, is the number of ways to partition $o_{i}$ colors among $r$ subplacements such that subplacement $p_{i}$ receives $o_{i j}$ of these colors. Altogether, this counts the number of ways to color the figures of the subplacements $p_{i}$ as to obtain a valid set of subplacements $\bar{p}_{i}$ that decolor to $p_{i}$. It follows that $f_{S}(n) \prod_{i=1}^{\mu} c_{i} !=f_{\bar{S}}(n)$, as desired.

\section{Intersection Schemas}

In the previous section, we showed that we can write the function $f_{S}(n)$ in the form given in equation (6). In particular, we have that

$$
f_{S}(n)=\sum_{r=1}^{m} \sum \frac{1}{r !} a\left(g_{1}\right) a\left(g_{2}\right) \cdots a\left(g_{r}\right) N^{r}
$$

where the function $a$ is given as in equation (7).

In this section, we will prove that a large class of functions written in this form give rise to polynomial sequences of binomial type. To do this, we will define an object called an intersection schema, which will generalize many of the properties of overlap graphs we encountered in the previous section.

Definition 16. A weighted set is a set $S$ (either finite or infinite) along with a weight function $w: S \rightarrow \mathbb{Z}^{+}$(from $S$ to the positive integers) such that for any $W$, there are only finitely many elements $x$ of $S$ such that $w(x) \leqslant W$.

Definition 17. Given a weighted set $S$, the set of $S$-labeled graphs is the set of graphs where each vertex is labelled by an element in $S$. We shall denote this set as $L G(S)$. We define the weight $w(g)$ of an element $g$ of $L G(S)$ to simply be the sum of the weights of its labels. We will also denote the subset of $L G(S)$ consisting of connected $S$-labeled graphs as $L C G(S)$. Note that we consider two graphs in $L G(S)$ to be equivalent if they are equivalent under a graph isomorphism that sends labeled vertices to similarly labeled vertices. 
Definition 18. An intersection schema is a weighted set $S$ along with a function $a$ : $L C G(S) \rightarrow \mathbb{C}$. Given an intersection schema, we define the polynomial $q_{i}(N)$ as:

$$
q_{i}(N)=\sum_{r=1}^{\infty} \sum \frac{1}{r !} a\left(g_{1}\right) a\left(g_{2}\right) \cdots a\left(g_{r}\right) N^{r}
$$

where the inner sum runs over all ordered $r$-tuples $\left(g_{1}, g_{2}, \ldots, g_{r}\right)$ of elements of $L C G(S)$ such that $\sum_{j=1}^{r} w\left(g_{j}\right)=i$. By default, we set $q_{0}(N)=1$.

Finally, we will need the following binomial identity:

Lemma 19. We have that

$$
n^{k}=\sum_{r=1}^{\infty} \sum_{m_{1}+\cdots+m_{r}=k} \frac{k !}{m_{1} ! m_{2} ! \cdots m_{r} !}\left(\begin{array}{l}
n \\
r
\end{array}\right)
$$

where the inner sum runs over all compositions of $k$.

Proof. The left hand side is the number of ways to color a set of $k$ items with $n$ colors. The right hand side counts this same number; here the $m_{i}$ correspond to sizes of sets of items colored the same color, the first multinomial coefficient corresponds to the number of ways to distribute the $k$ items into these groups of size $m_{i}$, and the binomial coefficient corresponds to the number of ways to choose $r$ colors for these $r$ groups out of the total $n$ colors.

We can now state and prove our main theorem.

Theorem 20. Let $\mathcal{I}$ be an intersection schema. Then

$$
\left(\sum_{i=0}^{\infty} q_{i}(1) x^{i}\right)^{N}=\left(\sum_{i=0}^{\infty} q_{i}(N) x^{i}\right)
$$

Proof. Let

$$
F(x)=\sum_{i=0}^{\infty} q_{i}(1) x^{i}
$$

and let

$$
F_{N}(x)=\sum_{i=0}^{\infty} q_{i}(N) x^{i}
$$

By the formula for $q_{i}(n)$ given in Definition 18, we can rewrite $F(x)$ as

$$
F(x)=1+\sum_{r=1}^{\infty} \sum \frac{1}{r !} a\left(g_{1}\right) a\left(g_{2}\right) \ldots a\left(g_{r}\right) x^{w\left(g_{1}\right)+w\left(g_{2}\right)+\cdots+w\left(g_{r}\right)}
$$


where the inner sum runs over all ordered $r$-tuples $\left(g_{1}, g_{2}, \ldots, g_{r}\right)$ of elements of $L C G(S)$.

We will now show by comparing terms that $F(x)^{N}=F_{N}(x)$. For sake of convenience, we will assume that the $a(g)$ are arbitrary non-commuting variables; i.e. that we do not necessarily have $a\left(g_{1}\right) a\left(g_{2}\right)=a\left(g_{2}\right) a\left(g_{1}\right)$ (of course, since $a\left(g_{i}\right) \in \mathbb{C}$, they do commute, but we will remove this restriction for now).

A general term in $F_{N}(x)$ looks like

$$
\frac{N^{r}}{r !} a\left(g_{1}\right) a\left(g_{2}\right) \ldots a\left(g_{r}\right) x^{w\left(g_{1}\right)+w\left(g_{2}\right)+\cdots+w\left(g_{r}\right)} .
$$

We will show that the coefficient of $a\left(g_{1}\right) a\left(g_{2}\right) \ldots a\left(g_{r}\right) x^{w\left(g_{1}\right)+w\left(g_{2}\right)+\cdots+w\left(g_{r}\right)}$ in $F(x)^{N}$ is also $\frac{N^{r}}{r !}$, thus completing the proof. To see this, first note that since we are assuming the $a(g)$ s do not commute, the terms in $F(x)$ which could contribute to this coefficient in $F(x)^{N}$ are of the form

$$
\frac{1}{j !} a\left(g_{i}\right) a\left(g_{i+1}\right) \ldots a\left(g_{i+j-1}\right) x^{w\left(g_{i}\right)+w\left(g_{i+1}\right)+\cdots+w\left(g_{i+j-1}\right)}
$$

(in other words, consecutive blocks of $a\left(g_{i}\right) \mathrm{s}$ ). But now, it follows directly from expansion that the coefficient of

$$
a\left(g_{1}\right) a\left(g_{2}\right) \ldots a\left(g_{r}\right) x^{w\left(g_{1}\right)+w\left(g_{2}\right)+\cdots+w\left(g_{r}\right)}
$$

in $F(x)^{N}$ is equal to

$$
\sum_{s} \sum_{m_{1}+\cdots+m_{s}=r} \frac{1}{m_{1} ! m_{2} ! \ldots m_{s} !}\left(\begin{array}{c}
N \\
s
\end{array}\right)
$$

which by Lemma 19 is simply equal to $\frac{N^{r}}{r !}$, as desired. (The ordered partitions arise from the different ways to divide the product $a\left(g_{1}\right) a\left(g_{2}\right) \ldots a\left(g_{r}\right)$ into consecutive "blocks").

We can now directly apply this theorem about intersection schemas to the case of non-overlapping placements.

Theorem 21. Let $S$ be a (possibly infinite) set of connected d-dimensional figures, and let $p_{k}\left(n^{d}\right)$ be the number of ways to place some collection of these figures (possibly using the same figure in $S$ repeatedly) that have a total of $k$ edges on $T_{n}^{d}$. Then the $p_{k}\left(n^{d}\right)$ are eventually polynomials in $n^{d}$, and these polynomials form a sequence of binomial type.

Proof. Define the following intersection schema. Our weighted set $S$ is just the set $S$ of $d$-dimensional figures, where the weight of a figure is simply its number of edges. The function $a$ is defined as in equation (7); note that graphs in $L C G(S)$ are just connected overlap graphs for some set of figures. Then Theorem 15 shows that for each $k, p_{k}\left(n^{d}\right)$ is eventually equal to $q_{k}\left(n^{d}\right)$. Our main theorem about intersection schemas (Theorem 20) then shows that the polynomials $q_{k}\left(n^{d}\right)$ form a sequence of binomial type, as desired. 


\section{Generalizations}

Up until now, this paper has been concerned only with placing $d$-dimensional figures on $d$-dimensional toroidal grid graphs. However, the machinery of intersection schemas and inclusion-exclusion on overlap graphs allow us to prove a much wider range of results. In fact, it seems that any problem involving placing finite non-overlapping collections of subgraphs on larger and larger periodic graphs gives rise to an eventual polynomial sequence; in addition, if the underlying periodic graphs are (in some sense) "toroidal", then this polynomial sequence is of binomial type. In this section, we will consider some generalizations of Theorems 5 and 21 that capture this idea.

\subsection{Other weights}

In the proof of Theorem 21, we assigned the weight of a figure to be its number of edges. However, since Theorem 20 works for any valid weight function, we can essentially assign whatever weights we want to figures (as long as not too many figures have small weight). We can formalize this in the following statement.

Theorem 22. Let $S$ be a (possibly infinite) set of connected d-dimensional figures, and let $w$ be a function from $S$ to $\mathbb{Z}^{+}$such that for any $x$, there are only finitely many figures $f \in S$ such that $w(f)=x$. Let $p_{k}\left(n^{d}\right)$ be the number of ways to place some collection of these figures (possibly using the same figure in $S$ repeatedly) that have a total of $k$ edges on $T_{n}^{d}$. Then the $p_{k}\left(n^{d}\right)$ are eventually polynomials, and these polynomials form a sequence of binomial type.

For example, all the following polynomial sequences are polynomial sequences of binomial-type:

- Let $p_{k}\left(n^{2}\right)$ be the number of ways to place $a$ L-shaped triominos and $b$ T-shaped pentominos on an $n \times n$ toroidal grid such that $7 a+2 b=k$. Then $p_{k}\left(n^{2}\right)$ is eventually a polynomial sequence of binomial type.

- Let $p_{k}\left(n^{d}\right)$ be the number of ways to place some number of $d$-dimensional figures on a $d$-dimensional toroidal grid graph such that the sum of the squares of the number of edges over all figures equals $k$. Then $p_{k}\left(n^{d}\right)$ is eventually a polynomial sequence of binomial type.

- Let $p_{k}\left(n^{d}\right)$ be the number of ways to place some number of $d$-dimensional figures on a $d$-dimensional toroidal grid graph such that the total number of edges in the figures equals $k$, and then to color each figure that has at least 3 edges with one of 50 colors. Then $p_{k}\left(n^{d}\right)$ is eventually a polynomial sequence of binomial type. 


\subsection{Continuous variant}

We can easily adapt intersection schemas to handle a continuous variant of our problem. To do this, we replace the $d$-dimensional toroidal grid graph $T_{n}^{d}$ with a continuous $d$ dimensional torus of side length $n$, and the concept of a $d$-dimensional figure with a bounded measurable set in $d$-dimensional Euclidean space. Then instead of counting the number of ways to place some number of objects so that they do not overlap, we instead consider the total measure of non-overlapping placements in state space.

For the case where our collection of figures contains only one object, we have the following nice probabilistic result.

Theorem 23. Let $\mathcal{S}$ be a bounded measurable set in d-dimensional Euclidean space. Let $p_{k}\left(n^{d}\right)$ be the probability that no two copies intersect when we place $k$ copies of $\mathcal{S}$ independently and uniformly at random inside a d-dimensional torus of side-length $n$. Then $n^{d k} p_{k}\left(n^{d}\right)$ is eventually a polynomial for each $k$, and these polynomials form a sequence of binomial-type.

\section{$5.3 \quad$ Non-toroidal grids}

We can also ask what happens if, instead of placing our figures on the toroidal grid graph $T_{n}^{d}$, we place them on the regular grid graph $L_{n}^{d}$. It turns out that in this case we lose the binomial-type property. However, the number of possible placements is still a polynomial (in $n$ instead of $n^{d}$, however), and we therefore have the following analogue to Theorem 5 .

Theorem 24. Let $S$ be a finite multiset of d-dimensional figures. Let $f_{S}(n)$ be the number of ways to place all of the figures in $S$ on $L_{n}^{d}$ such that none overlap. Then there exists a positive integer $n_{0}$ and a polynomial $p(x)$ such that $f_{S}(n)=p(n)$ for all $n \geqslant n_{0}$.

Proof. We follow the proof of Theorem 5, with the slight change that for each configuration, instead of there being $n^{d}$ valid translations, there are only $\left(n-g_{1}\right)\left(n-g_{2}\right) \ldots\left(n-g_{d}\right)$ valid translations, where $g_{i}$ is the girth of the configuration in dimension $i$ (that is, $\left.g_{i}(c)=\max _{x, y \in c}\left|x_{i}-y_{i}\right|\right)$.

We can extend this even farther. By the same reasoning as in the proof of Theorem 5 , the above result holds for grid "rectangles" with unequal dimensions (like $n \times 2 n$ rectangles, or $4 n \times 5 n \times 6 n$ boxes). In fact, we have the following general result:

Theorem 25. Let $G$ be a graph formed by taking a finite subset of the unit d-dimensional cells comprising $T_{\infty}^{d}$. Let $G^{n}$ be the graph obtained by replacing each unit cell by a cell of length $n$ divided regularly into $n^{d}$ unit cells. Let $S$ be a finite multiset of d-dimensional figures, and let $f_{S}(n)$ be the number of ways to place all of the figures in $S$ on $G^{n}$ such that none overlap. Then there exists a positive integer $n_{0}$ and a polynomial $p(x)$ such that $f_{S}(n)=p(n)$ for all $n \geqslant n_{0}$. 
Proof. Again, by following the same reasoning in 5, it suffices to show that the number of ways to place any one figure on $G^{n}$ is a polynomial in $n$.

To show this, call our figure $f$, and divide $G^{n}$ into copies of $L_{n}^{d}$ (in the same way that we can divide $G^{1}$ into unit $d$-dimensional cells). The number of ways to place $f$ in $L_{n}^{d}$ is a polynomial in $n$ (namely, the same polynomial used in the proof of Theorem 24 above), so the total number of ways to place $f$ so that it stays entirely within one of these copies of $L_{n}^{d}$ is also a polynomial in $n$. Now, by similar reasoning, the number of ways this figure can intersect exactly $c$ of these $n$-dimensional cells is a polynomial in $n$ (since for each specific choice of $c$ cells, the number of ways this figure can intersect exactly those cells will be a polynomial in $n$ ). By summing all of these polynomials (and there are a finite number of these, since $G$ contains a finite number of unit cells), we find that the total number of ways to place $f$ in $G^{n}$ is a polynomial in $n$, as desired.

\subsection{Other lattices}

Finally, the only discrete lattice we have considered is the square lattice. However, analogues of all of the above theorems exist for other lattices, such as triangular lattices and hexagonal lattices (and by exactly the same logic).

\section{Application to Chromatic Polynomials}

The following open problem appears as Exercise 4.82 in Enumerative Combinatorics, vol. 1.

Theorem 26. Let $\chi_{n}(x)$ be the chromatic polynomial of the $n \times n$ toroidal grid graph, and let $q_{k}\left(n^{2}\right)$ be the coefficient of $x^{n^{2}-k}$ in $\chi_{n}(x)$. Then $(-1)^{k} q_{k}\left(n^{2}\right)$ is eventually a polynomial in $n^{2}$, and this sequence of polynomials is of binomial-type.

In this section, we will provide a proof of this theorem, thus resolving this open problem. In addition, we will prove that the above claim holds not just for the $n \times n$ toroidal grid graph but for $T_{n}^{d}$, for any number of dimensions $d$.

To do this, we will reduce the problem of computing the coefficient of $x^{n^{2}-k}$ in $\chi_{n}(x)$ to a placement problem, and then apply Theorem 21. Our main tool for doing this will be Whitney's broken-circuit theorem, stated below.

Definition 27. In a graph $G=(V, E)$ with a total ordering on the edges, a broken circuit is a subset of $E$ formed by taking a cycle in $G$ and removing the largest edge (with respect to the ordering).

Theorem 28. (Whitney's broken-circuit theorem) Let $G$ be a finite graph with a strict ordering on the edge set $E$. Then, for $n$ between 0 and $|V|$ inclusive, the coefficient of $\lambda^{|V|-k}$ in $\chi_{G}(\lambda)$ is equal to $(-1)^{k}$ times the number of $k$-element subsets of $E$ which do not contain any broken-circuit of $G$ as a subset.

Proof. See [11]. 
It would be ideal if we could choose as our set $S$ of figures the set of connected $d$-dimensional figures which do not contain any broken-circuits. Unfortunately, the definition of broken-circuit depends on the ordering of the edges in the graph. Fortunately, we can choose an ordering of edges on $T_{n}^{d}$ that largely remedies this problem.

Definition 29. In the graph $T_{n}^{d}$, we call a total ordering of the edge set $E$ natural if it satisfies the following properties:

1. Each segment parallel to $u_{i}$ (where $u_{i}$ is the unit vector in dimension $i$ ) for $i \geqslant 2$ occurs before all edges parallel to $u_{1}$ (call these edges horizontal).

2. If a horizontal edge $e$ connects points $\left(x_{1}, x_{2}, \ldots, x_{n}\right)$ and $\left(x_{1}+1, x_{2}, \ldots, x_{n}\right)$, let the projection of edge $e, p(e)$, be the $(n-1)$-tuple $\left(x_{2}, x_{3}, \ldots, x_{n}\right)$. To compare two horizontal edges $e_{1}$ and $e_{2}$, let the larger edge be the edge with the lexicographically later projection vector.

A natural ordering of the edge set of the graph $T_{\infty}^{d}$ is defined in the same way.

For the remainder of this paper, for each of the graphs $T_{n}^{d}$ and $T_{\infty}^{d}$, fix a natural edge ordering; we will refer to this ordering below as "the natural edge ordering".

Definition 30. We say a figure is locally good if its embedding in $T_{\infty}^{d}$ contains no brokencircuit under the natural edge ordering (note that if a translate of some subset of $T_{\infty}^{d}$ contains a broken-circuit iff the subset contains a broken-circuit, by construction of the natural edge ordering). We say that the placement of a figure in $T_{n}^{d}$ is globally good if the corresponding subset of $T_{n}^{d}$ contains no broken-circuit under the natural edge ordering. If a figure is not locally/globally good, then it is locally/globally bad.

Now, we can let $S$ be the set of all locally good figures. However, note that it is possible to place a figure that is locally good on $T_{n}^{d}$ such that it is globally bad (for example, for $d=2$, we can achieve this in certain cases by placing it so that it intersects the vertical line $x_{2}=n$ ). Similarly, it is possible to place a figure which is locally bad on $T_{n}^{d}$ so that it is globally good. The following theorem will allow us to ignore such cases.

Theorem 31. The total number of ways to place a globally bad cycle-free figure with $k$ edges on $T_{n}^{d}$ (over all possible figures with $k$ edges) is equal to the number of ways to place a locally bad cycle-free figure with $k$ edges on $T_{n}^{d}$.

Proof. We will exhibit a bijection between these two sets. Assume we have a figure $f$ (with $|E|=k$ ) which is cycle-free but globally bad. Since it is globally bad, it must contain some number of broken-circuits (under the natural edge ordering for $T_{n}^{d}$ ). Let the number of broken-circuits be $b$, and let $e_{i}$ be the edge needed to make the $i$ th broken circuit a cycle. Note first that we cannot have $e_{i}=e_{j}$ for $i \neq j$, because if this were the case, then there would be two distinct paths between the endpoints of $e_{i}$ in $f$, which would imply that there is a cycle in $f$. Thus the $e_{i}$ comprise $b$ different edges.

Let $\bar{f}$ be the graph formed by adding all of these edges to $f$ (so $\bar{f}$ now has $k+b$ edges). Now, consider $\bar{f}$ as a subgraph of $T_{\infty}^{d}$ with its natural edge ordering. Let $f^{\prime}$ be 
the minimum spanning tree of $\bar{f}$, where we let the weight of the $r$ th largest edge of $\bar{f}$ be $r$. We now claim that $f^{\prime}$ is locally bad (it is cycle-free since it is a tree). To see this, note first that since $f^{\prime}$ and $f$ are both spanning trees of $\bar{f}$, they both must have the same number $k$ of edges. Next, let $e_{1}^{\prime}, e_{2}^{\prime}, \ldots, e_{b}^{\prime}$ be the $b$ edges belonging to $\bar{f}$ but not to $f^{\prime}$. Note that (by the properties of minimum spanning trees) if we add in $e_{i}^{\prime}$ for any $i$, we will construct a unique simple cycle; moreover (again by the properties of minimum spanning trees), $e_{i}^{\prime}$ will have the heaviest weight in this cycle. This implies that this set of edges in $f^{\prime}$ (minus $e_{i}^{\prime}$ ) forms a broken circuit under the local natural edge ordering, so $f^{\prime}$ is locally bad (and in fact, it contains $b$ broken-circuits under this edge ordering).

This procedure is a map which sends placements of globally bad cycle-free figures $f$ with $k$ edges to placements of locally bad cycle-free figures $f^{\prime}$ with $k$ edges. Now, note that we can invert this map via the following procedure, thus showing that this map is a bijection. As before, we take the $b$ broken-circuits and the $b$ edges $e_{i}^{\prime}$ required to make the $i$ th broken circuit a cycle. We then add these $b$ edges to $f^{\prime}$ to construct $\bar{f}$, and once we do this we let $f$ be the minimum spanning tree of $\bar{f}$ with respect to the natural edge ordering of $T_{n}^{d}$. To see that this restores the original $f$, note first that the figure $\bar{f}$ constructed in going from $f$ to $f^{\prime}$ contains exactly the same edges as the figure $\bar{f}$ constructed in going back from $f^{\prime}$ to $f$. Next, note that none of the edges $e_{i}$ can belong to the minimum spanning tree of $\bar{f}$ with respect to the natural edge ordering of $T_{n}^{d}$; this is since each such edge $e_{i}$ is the largest edge in a cycle, and such edges never occur in minimum spanning trees. But since $\bar{f}$ has $k+b$ edges, and there are $b$ edges $e_{i}$, this must mean that this minimum spanning tree is exactly $f$, as desired.

Corollary 32. The total number of ways to place a locally good cycle-free figure with $k$ edges on $T_{n}^{d}$ (over all possible figures with $k$ edges) is equal to the number of ways to place a globally good cycle-free figure with $k$ edges on $T_{n}^{d}$.

Proof. Consider the following four sets of possible placements of figures with $k$ edges: $S_{G G}$, the set of locally good and globally good placements, $S_{G B}$, the set of locally good but globally bad placements, $S_{B G}$, the set of locally bad but globally good placements, and $S_{B B}$, the set of locally bad and globally bad placements. We wish to show that $\left|S_{G G}\right|+\left|S_{G B}\right|=\left|S_{G G}\right|+\left|S_{B G}\right|$, or equivalently, that $\left|S_{G B}\right|=\left|S_{B G}\right|$.

To do this, it suffices to show that $\left|S_{G B}\right|+\left|S_{B B}\right|=\left|S_{B G}\right|+\left|S_{B B}\right|$. Let $C$ be the set of placements of a figure with $k$ edges that has a cycle; note that any such placement must be both locally bad and globally bad, since any graph with a cycle contains a broken-circuit under any edge-ordering. We thus have that $C \subset S_{B B}$. Because of this, Theorem 31 implies that $\left|S_{G B}\right|+\left|S_{B B}\right|-|C|=\left|S_{B G}\right|+\left|S_{B B}\right|-|C|$, and therefore that $\left|S_{G B}\right|+\left|S_{B B}\right|=\left|S_{B G}\right|+\left|S_{B B}\right|$, as desired.

We can now prove the following generalization of Theorem 26.

Theorem 33. Fix $d$, and let $\chi_{n}(x)$ be the chromatic polynomial of $T_{n}^{d}$. Let $q_{k}\left(n^{d}\right)$ be the coefficient of $x^{n^{d}-k}$ in $\chi_{n}(x)$. Then $(-1)^{k} q_{k}\left(n^{d}\right)$ is eventually a polynomial in $n^{d}$, and this sequence of polynomials is of binomial-type. 
Proof. By Whitney's broken-circuit theorem, $q_{k}\left(n^{d}\right)$ is equal to $(-1)^{k}$ times the number of $k$-element subsets of $T_{n}^{d}$ which contain no broken-circuit. By choosing a natural edgeordering for $T_{n}^{d}$ and using the notation above, $(-1)^{k} q_{k}\left(n^{d}\right)$ is just the number of ways to place a globally good figure with $k$ edges on $T_{n}^{d}$. By Corollary 32 , this is equal to the number of ways to place a locally good figure with $k$ edges on $T_{n}^{d}$. By choosing $S$ to be the set of locally good connected figures, it follows from Theorem 21 that this number is indeed a polynomial in $n^{d}$ and that these polynomials form a sequence of binomial-type, as desired.

\section{Acknowledgements}

This research was performed as part of MIT's Undergraduate Research Opportunities Program (UROP) in the summer of 2011. The author would like to thank Prof. Richard Stanley for introducing him to this problem and mentoring him over the course of this project.

\section{References}

[1] G. A. Baker, Linked-Cluster Expansion for the Graph-Vertex Coloration Problem, J. Comb. Theory 10 (1971), 217-231.

[2] R. J. Baxter, Exactly solved models in statistical mechanics, Academic Press, 1982.

[3] N. L. Biggs, Chromatic and thermodynamic limits, J. Phys. A 8 (1975), L110-L112.

[4] S.C. Chang, R. Shrock, General structural results for Potts partition functions on lattice strips, Phys. A 316 (2002), 335-379.

[5] S.C. Chang, R. Shrock, Tutte polynomials and related asymptotic limiting functions for recursive families of graphs, Adv. in Appl. Math. 32 (2004), 44-87.

[6] D. Kim, I.G. Enting, The limit of chromatic polynomials, J. Comb. Theory B 26 (1979), 327-336.

[7] E. H. Lieb, Residual entropy of square ice, Phys. Rev. 162 (1967), 162-172.

[8] J. F. Nagle, A new subgraph expansion for obtaining coloring polynomials for graphs, J. Comb. Theory 10 (1971), 42-59.

[9] R. P. Stanley, Enumerative Combinatorics, Cambridge University Press, Vol. 1, ed. 2, 2011.

[10] G.-C. Rota, D. Kahaner, A. Odlyzko, Finite Operator Calculus, J. Math. Anal. Appl. 42 (1973), 685-760.

[11] H. Whitney, A logical expansion in mathematics, Bull. Amer. Math. Soc. 38 (1932), 572-579. 